\title{
Multidimensional Instability and Dynamics of Spin Avalanches in Crystals of Nanomagnets
}

\author{
O. Jukimenko, ${ }^{1}$ C. M. Dion, ${ }^{1}$ M. Marklund, ${ }^{1,2}$ and V. Bychkov ${ }^{1}$ \\ ${ }^{1}$ Department of Physics, Umeå University, SE-901 87 Umeå, Sweden \\ ${ }^{2}$ Department of Applied Physics, Chalmers University of Technology, SE-412 96 Göteborg, Sweden
}

(Received 16 January 2014; published 21 November 2014)

\begin{abstract}
We obtain a fundamental instability of the magnetization-switching fronts in superparamagnetic and ferromagnetic materials such as crystals of nanomagnets, ferromagnetic nanowires, and systems of quantum dots with large spin. We develop the instability theory for both linear and nonlinear stages. By using numerical simulations we investigate the instability properties focusing on spin avalanches in crystals of nanomagnets. The instability distorts spontaneously the fronts and leads to a complex multidimensional front dynamics. We show that the instability has a universal physical nature, with a deep relationship to a wide variety of physical systems, such as the Darrieus-Landau instability of deflagration fronts in combustion, inertial confinement fusion, and thermonuclear supernovae, and the instability of doping fronts in organic semiconductors.
\end{abstract}

DOI: 10.1103/PhysRevLett.113.217206

PACS numbers: 75.50.Xx, 47.20.-k, 47.70.Pq, 75.60.Jk

Advanced magnetic materials with superparamagnetic and ferromagnetic properties, such as molecular (nano) magnets, ferromagnetic nanowires, and quantum dots with spins larger than $1 / 2$, are the focus of active research due to their promising applications to spintronics and quantum data storage [1-4]. In contrast to classical magnetic dipoles, nanomagnets may keep their spin orientation unchanged in altering magnetic fields $[1,5,6]$. Spontaneous transition of a nanomagnet from the metastable state (against the field) to the ground state (along the field) is hindered by the magnetic anisotropy. In crystals of nanomagnets, the transition may be induced by Zeeman energy release in a spin avalanche, spreading in the form of a magnetic deflagration front (due to thermal conduction) [7-13] or a magnetic detonation front (due to shock waves) [13-15]. Then, in an external magnetic field, a spin-avalanche front switches the magnetization of a crystal to the energetically favorable state, similar to the propagation of a domain wall in ferromagnetic nanowires [2,3].

So far, almost all experimental and theoretical studies of spin avalanches have assumed a simplified planar 1D geometry of the propagating fronts [7-13]. Only recently, the possibility of 3D bending of a spin-avalanche front has been encountered in heavy numerical simulations for the specific propagation mechanism controlled by the dipoledipole interaction close to the tunneling resonance [16]. We stress that the propagation mechanism studied in [16] is not related to the temperature gradient across the front, and thus conceptually different from the experimentally observed magnetic deflagration $[7,8,12]$. It was suggested in [16] that the $3 \mathrm{D}$ bending of the magnetization-switching front is a specific feature of the dipole-controlled propagation mechanism, and thus may suffer from a narrow domain of applicability. Moreover, the very existence of the dipole-controlled propagation mechanism studied in [16] has not yet been confirmed experimentally. Thus, the issue of multidimensional magnetic deflagration dynamics has remained open.

Here we demonstrate that the 3D bending of the magnetization-switching fronts in superparamagnetic or ferromagnetic materials is a universal physical phenomenon, arising in a common situation when the front propagation speed is controlled by the applied magnetic field. We find that the instability distorts such fronts and increases their propagation speed. We develop a theory for both the linear and nonlinear stages of the instability, and perform numerical simulations to investigate the instability properties, focusing on spin-avalanche fronts in crystals of nanomagnets. We demonstrate that the instability leads to a complex multidimensional dynamics, with the possibility of stationary cellular structures emerging at the fronts or powerful front acceleration. Among other conclusions, the present theory explains 3D bending of the dipole-controlled fronts encountered in the numerical simulations of Ref. [16] as a particular case. The universal approach to the problem used in our work makes it possible to understand the deep physical relation of the present instability to phenomena from other fields of physics such as the Darrieus-Landau instability of deflagration fronts in combustion, inertial confinement fusion, and thermonuclear supernovae [17-20], as well as the instability of doping fronts in organic semiconductors [21,22].

We consider a generic model of an initially planar front in a magnetically active material. The front propagation speed $U_{f}$ depends on the external magnetic field $\mathbf{B}$ applied normally to the original front; we set the $z$ axis along the direction of the external magnetic field. The front modifies the magnetic properties of the material. In crystals of nanomagnets, for the 2D geometry of Fig. 1, the magnetization 


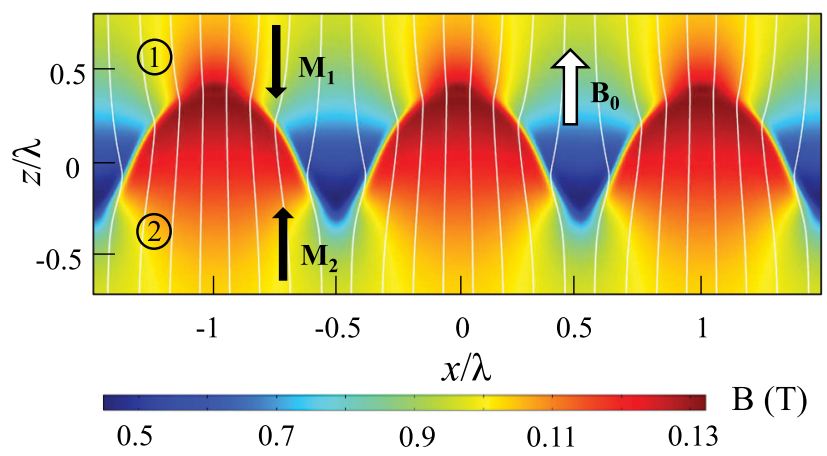

FIG. 1 (color online). The magnetic field (shown by colors and field lines) at a 2D curved stationary front propagating at constant speed as obtained using numerical simulations of Eqs. (4) and (5) for $\mu_{0} M=0.05 \mathrm{~T}, B_{0}=0.1 \mathrm{~T}$, and $\lambda / L_{f}=20$.

vector of a fixed absolute value $M$ switches from $\mathbf{M}_{1} \equiv$ $\left(M_{x 1} ; M_{z 1}\right)=(0 ;-M)$ ahead of the front (index 1 and label 1 in Fig. 1) to $\mathbf{M}_{2} \equiv\left(M_{x 2} ; M_{z 2}\right)=(0 ; M)$ behind the front (index 2 and label 2 in Fig. 1). Deviations of the magnetization vector from the $\pm z$ axis may be neglected [13].

The physical meaning of the instability may be understood from Fig. 1. The front bending modifies the magnetic field, with the absolute field value increasing close to the front humps in agreement with Maxwell's equations. This increase of the magnetic field is similar to the increase of the electric field by the convex parts of a conductor (e.g., at a distorted doping front $[21,22]$ ), or to modifications of the gas velocity at the humps of a wrinkled flame front [17]; here the magnetic, electric, and velocity fields play conceptually the same role. In turn, the increase of the magnetic field close to the front humps produces a local increase of the front speed, thus leading to further unstable growth of the hump. We demonstrate this effect below by solving the stability problem for the originally planar magnetic deflagration front $Z_{f}=U_{f} t$ with the initial magnetic field normal to the front, $\mathbf{B}_{0}=\hat{\mathbf{a}}_{z} B_{0}$. The front position is defined as $z=Z_{f}(\mathbf{x}, t)$.

We consider infinitesimal front perturbations as a superposition of Fourier modes, $Z_{f}(x, t)=U_{f} t+\tilde{Z}_{f}(\mathbf{x}, t)$, where $\tilde{Z}_{f}(\mathbf{x}, t)=\sum_{k} \tilde{Z}_{k} \exp (i \mathbf{k} \cdot \mathbf{x}+\sigma t)$ with the perturbation wave number $k=2 \pi / \lambda$, the wavelength $\lambda$, and the factor $\sigma$. The purpose of the linear stability problem is to find the dispersion relation $\sigma(k)$; the front is unstable with respect to the bending if $\operatorname{Re}(\sigma)>0$ for at least some values of $k$. As we show below, the factor $\sigma$ is real and positive in this problem, and may be called "the instability growth rate." We consider the stability of an infinitely thin front, $k L_{f} \ll 1$, where $L_{f}$ is the front thickness controlled by transport processes, e.g., by thermal diffusion $\kappa$ in the case of magnetic deflagration, $L_{f} \equiv \kappa / U_{f}$. Perturbations of the front induce perturbations of the magnetic field both ahead and behind the front, $\mathbf{B}=\mathbf{B}_{0}+\sum \tilde{\mathbf{B}}_{k}(z) \exp (i \mathbf{k} \cdot \mathbf{x}+\sigma t)$, which satisfy Maxwell's equations for a nonconducting medium, $\nabla \cdot \mathbf{B}=0, \nabla \times \mathbf{H}=0, \mathbf{B} / \mu_{0}=\mathbf{H}+\mathbf{M}$, where $\mu_{0}$ is the vacuum permeability. Taking into account the vanishing of the perturbations far away from the perturbed front, at $z \rightarrow \pm \infty$, we solve Maxwell's equations as $\tilde{\mathbf{B}}_{1,2}(z) \propto \exp (\mp k z)$. We match the solutions using the boundary conditions $\hat{\mathbf{a}}_{n} \cdot[\mathbf{B}]=0, \hat{\mathbf{a}}_{n} \times[\mathbf{H}]=0$, where the normal vector to the perturbed front is $\hat{\mathbf{a}}_{n}=\hat{\mathbf{a}}_{z}-\nabla_{\perp} Z_{f}$, within the linear problem, $\nabla_{\perp}$ corresponds to the transverse variables $\mathbf{x}$, and $[F] \equiv F_{2}-F_{1}$ designates the difference of any value $F$ across the front. After resolving the boundary conditions and Maxwell's equations, we find the relations between the field perturbations at the front, at $z=0$, and the front perturbations for any Fourier mode, $\tilde{B}_{z 1}=$ $\tilde{B}_{z 2}=\mu_{0} M k \tilde{Z}_{f}$, which reflects the increase of the magnetic field close to the perturbation humps, in agreement with Fig. 1. Within the linear stability problem, the perturbations of the front velocity are calculated as $\partial_{t} \tilde{Z}_{f}=U_{f}^{\prime} \tilde{B}_{z}$, with $U_{f}^{\prime} \equiv d U_{f} / d B$, and we find the dispersion relation, see Supplemental Material [23]

$$
\sigma=k U_{f}^{\prime} \mu_{0} M
$$

Thus, a thin magnetization-switching front is unconditionally unstable against multidimensional perturbations bending the front. The structure of the dispersion relation, $\sigma \propto k$, is mathematically similar to the Darrieus-Landau instability of a flame front encountered in combustion, astrophysics, and laser fusion [17-20], and to the instability of doping fronts in organic semiconductors $[21,22]$. The similarity of these dispersion relations implies complex multidimensional dynamics of magnetic fronts, analogous to flames, with the possibility of cellular and fractal structures emerging at the fronts $[17,18]$. Still, as we show below, the magnetic instability demonstrates also some unique features, such as powerful front acceleration, which does not happen for the traditional Darrieus-Landau instability.

The characteristic strength of the new instability $\sigma / U_{f} k$ is determined by the magnetization $M$ and the sensitivity of the front speed to the magnetic field perturbations $U_{f}^{\prime} / U_{f}$. In particular, in the case of Permalloy nanowires, the so-called "viscous" (i.e. controlled by dissipations) regime of domain-wall propagation corresponds to a front speed proportional to the applied field, $U_{f} \propto H$, with the proportionality factor about $1.1 \mathrm{~m}^{2} / \mathrm{sA}$ (see Ref. [2]). Taking $\mu_{0} M=1 \mathrm{~T}$ for a Permalloy and typical domain wall speed of $U_{f} \sim 500 \mathrm{~m} / \mathrm{s}$, we obtain an extremely strong instability with $\sigma / U_{f} k \sim 10^{3}$. In that case even minor front bending modifies the magnetic field strongly, with a considerable increase of the propagation speed of the domain wall.

In contrast to ferromagnetic materials, the magnetization of crystals of nanomagnets is rather moderate, corresponding to $\mu_{0} M \approx 0.05 \mathrm{~T}$ [24]. We take the dependence of the magnetic deflagration speed $U_{f}$ on the applied magnetic field from the experimental work in Ref. [8], shown by markers on Fig. 2 and fitted by the red curve. The curve 


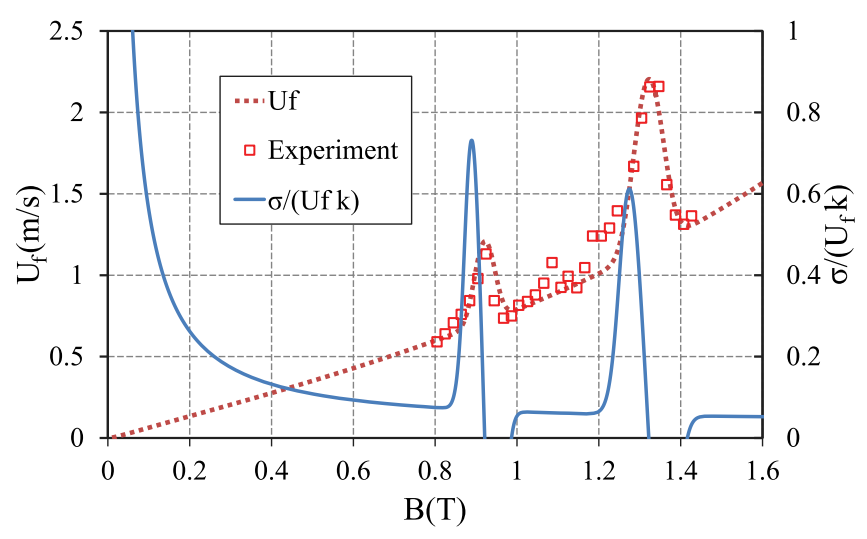

FIG. 2 (color online). The magnetic deflagration speed $U_{f}$ and the scaled instability growth rate, $\sigma / U_{f} k$, vs magnetic field, with $k=2 \pi / \lambda$, for magnetization $\mu_{0} M=0.05 \mathrm{~T}$. The markers present the experimental data for $U_{f}$ obtained in [8]. The parameters of the resonance peaks at $B_{1,2}=0.92 ; 1.32(\mathrm{~T})$ are $a_{1,2}=1.89 ; 2.61$ and $b_{1,2}=840 ; 870$.

reflects a monotonic increase of the magnetic deflagration speed with the field but for local peaks of $U_{f}$ due to quantum resonances at $B \approx 0.92 \mathrm{~T}$ and $1.3 \mathrm{~T}$. There are more resonances in the dependence, which have not been measured in Ref. [8], but may be found, e.g., in Ref. [12]. The monotonic part of the dependence may be described by a simple formula originating from combustion theory $[9,10,13]$, as $U_{f}=\sqrt{\kappa / \tau_{R} \mathrm{Ze}} \exp \left(-E_{a} / 2 T_{f}\right)$, where $\tau_{R}$ is the characteristic time of spin flipping and $\mathrm{Ze} \sim E_{a} / 4 T_{f}$ is the Zeldovich number. The activation energy $E_{a}$ (in temperature units) and temperature $T_{f}$ behind the front are determined by the applied magnetic field; see $[9,10,13]$ for details. In the present work we describe the resonances in $U_{f}$ by taking $\tau_{R}$ as a function of the magnetic field with the local resonance peaks approximated by the Gaussian function: $\tau_{R}=\tau_{0} /\left\{1+\Sigma a_{i} \exp \left[-b_{i}\left(B / B_{i}-1\right)^{2}\right]\right\}$, where $B_{i}$ is the respective resonance field, and the parameters $a_{i}, b_{i}$ control the height and width of the resonance. A similar Lorentzian shape of the resonance peaks has been suggested in the analytical model [25]. We take the resonance width and height as free parameters of the problem and calculate the relative instability strength $\sigma / U_{f} k$ in crystals of nanomagnets as presented in Fig. 2. In the chosen magnetic field domain, we observe three regions of considerable instability strength $\sigma / U_{f} k \sim 1$ : at low magnetic fields $B<0.4 \mathrm{~T}$ when the front velocity is small, and close to the resonances, when the front velocity is sensitive to the field perturbations.

As the amplitude of front perturbations grows, nonlinear effects become important with a possible saturation of the instability growth to a stationary (i.e. time-independent) cellular structure. We here solve the nonlinear problem of a stationary cellular front propagating with constant speed by using the classical Layzer model, which has been employed successfully within the theory of the Rayleigh-Taylor instability [18]. To be particular, in the nonlinear problem we consider an axisymmetric pattern of the curved front, which reproduces the most important quantitative properties of the respective 3D geometry, and still retains quasi2D simplifications from the analytical and numerical points of view. For comparison, it has been demonstrated that velocity increase of a curved Darrieus-Landau unstable flame is practically independent of a particular 3D or axisymmetric front shape [26]. Within the Layzer model, the magnetic field is approximated by the leading Fourier modes ahead of and behind the front, which are matched at the tip of the curved stationary front, see Supplemental Material [23]. Specifically, we consider the axisymmetric cell geometry shown in Fig. 3 and take the magnetic field in the form $\mathbf{B}_{0}+\tilde{\mathbf{B}}_{1,2}$, with $\tilde{\mathbf{B}}=-\nabla \phi$, the scalar potential $\phi=\Phi_{1,2} \exp (\mp k z) J_{0}(k r)$ and the zero-order Bessel function $J_{0}$. The amplitudes $\Phi_{1,2}$ are determined by the boundary conditions at the bent front. The front shape at the tip is parabolic, $Z(r)=-\alpha r^{2}$, where the coefficient $\alpha$ has to be found from the problem solution. By substituting the obtained magnetic field into the boundary conditions, we find an increase of the field at the front tip, $\tilde{B}_{z}(0) \equiv \tilde{B}_{0}=8 M \mu_{0} \alpha / k$, so that the front tip propagates at an increased speed $U_{f}\left(B_{0}+\tilde{B}_{0}\right)$. Since all points of a stationary front propagate at the same speed, we arrive at the equation

$$
U_{f}\left[B_{0}+\tilde{B}_{0} J_{0}(k r) \exp (k z)\right]=\hat{\mathbf{a}}_{z} \cdot \hat{\mathbf{a}}_{n} U_{f}\left(B_{0}+\tilde{B}_{0}\right),
$$

where $\hat{\mathbf{a}}_{n}$ is a normal vector to the front surface at $Z(r)$. Expanding Eq. (2) at the front tip in $k z \ll 1, k r \ll 1$, we obtain

$$
\tilde{B}_{0}=\frac{8 \mu_{0}^{2} M^{2} U_{f}^{\prime}}{U_{f}-4 \mu_{0} M U_{f}^{\prime}},
$$

where $U_{f}$ and $U_{f}^{\prime}$ are taken at $B_{0}+\tilde{B}_{0}$. The solution to Eq. (3) determines the increase of the magnetic field at the (a)

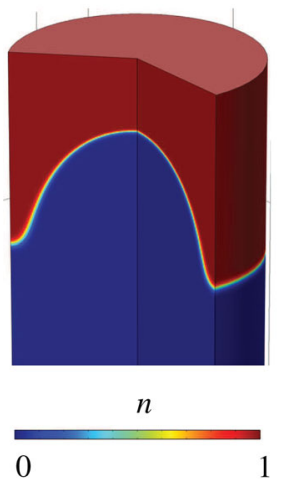

(b)

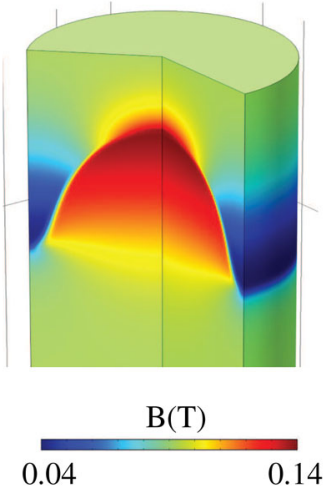

FIG. 3 (color online). A stationary curved magnetic deflagration front in the axisymmetric geometry obtained numerically using Eqs. (4) and (5) for the external magnetic field $B_{0}=0.1 \mathrm{~T}$, magnetization $\mu_{0} M=0.05 \mathrm{~T}$, and the scaled channel radius $R / L_{f}=21$. (a) Fraction of molecules in the metastable state, $n$. (b) Magnetic field magnitude. 
front tip, $\tilde{B}_{0}$, and hence the stationary front propagation speed. An important feature of Eq. (3) is the lack of a stationary solution for a sufficiently strong dependence of the front speed on the magnetic field, $4 \mu_{0} M U_{f}^{\prime} / U_{f}>1$. In that case, a powerful front acceleration with increasing curvature is expected with no saturation, until additional physical effects come into play and limit the front speed.

We have also validated the nonlinear theory by direct numerical simulations of the magnetic deflagration fronts for 2D and axisymmetric geometries using the basic equations of energy transfer and kinetics of spin flipping,

$$
\begin{gathered}
\frac{\partial E}{\partial t}=\nabla \cdot(\kappa \nabla E)-Q \frac{\partial n}{\partial t} \\
\frac{\partial n}{\partial t}=-\frac{1}{\tau_{R}} \exp \left(-\frac{E_{a}}{T}\right)\left[n-\frac{1}{\exp (Q / T)+1}\right],
\end{gathered}
$$

where $E$ is thermal (phonon) energy, $n$ is the fraction of nanomagnets in the metastable state, and $Q$ is the Zeeman energy release determined by the magnetic field at the front; see Refs. $[9,10,13]$ for details. Here $E, E_{a}$, and $Q$ are taken in temperature units. Equations (4) and (5) have been complemented by Maxwell's magnetostatic equations. The initial temperature was taken to be uniform and low, $T_{0}=0.1 \mathrm{~K}$, but for the small region close to the bottom of the computational domain, where it was raised to $T_{f}=$ $30 \mathrm{~K}$ required to induce the spin-flipping process. Slight bending of the hot region initiated the instability development. Boundaries of the computational domain are thermally insulating with $\hat{\mathbf{a}}_{n} \cdot \nabla T=0$; we also take $\hat{\mathbf{a}}_{n} \cdot \mathbf{B}=0$ at the side boundaries and uniform $B_{0}$ at the top or bottom of the domain.

Figures 1 and 3 show the characteristic shape of the curved stationary fronts obtained numerically as a result of the instability development for the $2 \mathrm{D}$ and axisymmetric geometries, respectively. Similar to stationary corrugated flames and doping fronts, the cellular multidimensional structure of the spin-avalanche fronts may be described as smooth humps facing the initial cold material and sharp cusps pointing at the transformed matter behind the front. The numerical modeling demonstrates also a strong increase of the magnetic field at the smooth tip, and a decrease of the field at the cusps, in agreement with the presented theory. In the numerical solution, we have also reproduced the regime of powerful acceleration for the cases of strong dependence of the front speed on the magnetic field close to the quantum resonances. In particular, for the first quantum resonance field $B_{1}=0.92 \mathrm{~T}$ in Fig. 2, with the width of the peak set by the parameter $b_{1}=840$ similar to the experimental data [8], the regime of powerful acceleration takes place for an applied field $B_{0}>0.84 \mathrm{~T}$. In this regime, the Huygens nonlinear stabilization of the front bending, which is common for flames [18], cannot stop the development of the instability, and the

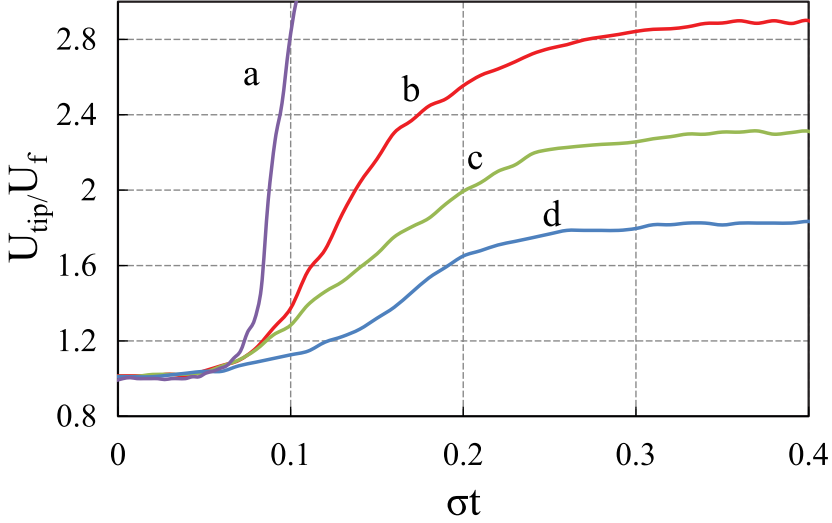

FIG. 4 (color online). Scaled velocity of the front tip, $U_{\text {tip }}$, versus scaled time for $B_{0}=0.86 \mathrm{~T}$ close to the first resonance for $b_{1}=840$ and $a_{1}=30$ (a); $a_{1}=10$ (b); $a_{1}=5$ (c); $a=1.89$ (d).

magnetic deflagration accelerates until the tip speed reaches the limiting speed characteristic for the quantum resonance peak. Here we stress that acceleration of this type is a unique feature of the magnetization fronts; the Darrieus-Landau instability in combustion, laser plasma, or astrophysics does not exhibit any effect of this kind. Figure 4 shows the acceleration of the spin avalanche close to the magnetic resonance with the resonance heights set by the parameter $a_{1}=1.89 ; 5 ; 10 ; 30$; the value $a_{1}=1.89$ stems from the experimental data [8]. At the same time, the theoretical model [24] of the quantum resonances suggests an ultimately large resonance height, well above the sound speed in the crystals, $2000 \mathrm{~m} / \mathrm{s}$. Then the instability may initiate a deflagration-to-detonation transition of magnetic avalanches, from the strongly subsonic speed of about $1 \mathrm{~m} / \mathrm{s}$ to the supersonic speed as observed in the nanomagnet experiments [14], and similar to the respective combustion process $[27,28]$.

Thus, the experimental signature of the obtained instability is the curved front shape and increased velocity of the magnetization front propagation. One may also expect that the present instability gives rise to an asymmetric shape of the resonance peaks; still, there is currently insufficient experimental data to test this expectation. Besides, the instability obtained in the present work may be responsible for the magnetic deflagration-to-detonation transition observed experimentally in Ref. [14]; the process of magnetic detonation triggering requires more studies.

To summarize, we have obtained a universal multidimensional instability of magnetization-switching fronts, which may develop spontaneously in superparamagnetic and ferromagnetic media such as crystals of nanomagnets, ferromagnetic nanowires and systems of quantum dots. The instability leads to a curved front structure with a possible strong increase of the propagation speed, and hence allows control of the front dynamics. Because of the universal 
instability properties, we expect our results to be applicable to a wide variety of problems.

The authors are grateful to the Swedish Research Council (VR) for financial support.

[1] L. Bogani and W. Wernsdorfer, Nat. Mater. 7, 179 (2008).

[2] Y. Nakatani, A. Thiaville, and J. Miltat, Nat. Mater. 2, 521 (2003).

[3] G. S. D. Beach, C. Nistor, C. Knutson, M. Tsoi, and J. L. Erskine, Nat. Mater. 4, 741 (2005).

[4] M. Misiorny, M. Hell, and M. R. Wegewijs, Nat. Phys. 9, 801 (2013).

[5] J. R. Friedman, M. P. Sarachik, J. Tejada, and R. Ziolo, Phys Rev. Lett. 76, 3830 (1996).

[6] L. Thomas, F. Lionti, R. Ballou, D. Gatteschi, R. Sessoli, and B. Barbara, Nature (London) 383, 145 (1996).

[7] Y. Suzuki, M. P. Sarachik, E. M. Chudnovsky, S. McHugh, R. Gonzalez-Rubio, N. Avraham, Y. Myasoedov, E. Zeldov, H. Shtrikman, N. E. Chakov, and G. Christou, Phys. Rev. Lett. 95, 147201 (2005).

[8] A. Hernández-Mínguez, J. M. Hernandez, F. Macià, A. García-Santiago, J. Tejada, and P. V. Santos, Phys. Rev. Lett. 95, 217205 (2005).

[9] D. A. Garanin and E. M. Chudnovsky, Phys. Rev. B 76, 054410 (2007).

[10] M. Modestov, V. Bychkov, and M. Marklund, Phys. Rev. B 83, 214417 (2011).

[11] S. McHugh, B. Wen, X. Ma, M. P. Sarachik, Y. Myasoedov, E. Zeldov, R. Bagai, and G. Christou, Phys. Rev. B 79, 174413 (2009).
[12] P. Subedi, S. Vélez, F. Macià, S. Li, M. P. Sarachik, J. Tejada, S. Mukherjee, G. Christou, and A. D. Kent, Phys. Rev. Lett. 110, 207203 (2013).

[13] C. M. Dion, O. Jukimenko, M. Modestov, M. Marklund, and V. Bychkov, Phys. Rev. B 87, 014409 (2013).

[14] W. Decelle, J. Vanacken, V. V. Moshchalkov, J. Tejada, J. M. Hernández, and F. Macià, Phys. Rev. Lett. 102, 027203 (2009).

[15] M. Modestov, V. Bychkov, and M. Marklund, Phys. Rev. Lett. 107, 207208 (2011).

[16] D. A. Garanin, Phys. Rev. B 88, 064413 (2013).

[17] C. K. Law, Combustion Physics (Cambridge University Press, Cambridge, England, 2006).

[18] V. Bychkov and M. Liberman, Phys. Rep. 325, 115 (2000).

[19] M. Modestov, V. Bychkov, D. Valiev, and M. Marklund, Phys. Rev. E 80, 046403 (2009).

[20] J. B. Bell, M. S. Day, C. A. Rendleman, S. E. Woosley, and M. Zingale, Astrophys. J. 606, 1029 (2004).

[21] V. Bychkov, P. Matyba, V. Akkerman, M. Modestov, D. Valiev, G. Brodin, C. K. Law, M. Marklund, and L. Edman, Phys. Rev. Lett. 107, 016103 (2011).

[22] V. Bychkov, O. Jukimenko, M. Modestov, and M. Marklund, Phys. Rev. B 85, 245212 (2012).

[23] See Supplemental Material at http://link.aps.org/ supplemental/10.1103/PhysRevLett.113.217206 for details of the linear and nonlinear stability analysis.

[24] D. A. Garanin and S. Shoyeb, Phys. Rev. B 85, 094403 (2012).

[25] M. N. Leuenberger and D. Loss, Phys. Rev. B 61, 1286.

[26] V. Bychkov and M. Liberman, Phys. Fluids 14, 2024 (2002).

[27] V. Bychkov, A. Petchenko, V. Akkerman, and L.-E. Eriksson, Phys. Rev. E 72, 046307 (2005).

[28] V. Bychkov, D. Valiev, and L.-E. Eriksson, Phys. Rev. Lett. 101, 164501 (2008). 